\title{
G

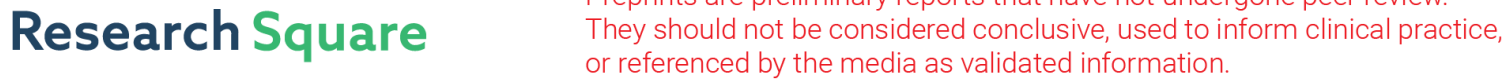 \\ Treatment of SARS-CoV-2 induced pneumonia with NAD+ in a mouse model
}

\section{Yisheng Jiang}

Institute of Genetics and Developmental Biology, Chinese Academy of Sciences https://orcid.org/00000003-3248-0152

\section{Yongqiang Deng}

Beijing Institute of Microbiology and Epidemiology

\section{Tiantian Ma}

Institute of Genetics and Developmental Biology, Chinese Academy of Sciences https://orcid.org/00000002-6677-2957

\section{Huanhuan Pang}

School of Pharmaceutical Sciences, Tsinghua University

\section{Zeping Hu}

Tsinghua University

\section{Cheng Qin}

Beijing Institute of Microbiology and Epidemiology https://orcid.org/0000-0002-0632-2807

\section{Zhiheng Xu ( $\nabla$ zhxu@genetics.ac.cn)}

Institute of Genetics and Developmental Biology, Chinese Academy of Sciences

\section{Article}

Keywords: Treatment, SARS-CoV-2, pneumonia with NAD+, mouse model

Posted Date: October 30th, 2020

DOI: https://doi.org/10.21203/rs.3.rs-96999/v1

License: (c) (i) This work is licensed under a Creative Commons Attribution 4.0 International License.

Read Full License 


\section{Abstract}

The global COVID-19 epidemic has spread rapidly around the world and has already caused the death of more than one million people. As there is yet no vaccine, it is urgent to develop effective strategies to treat COVID-19 patients. Here, we used a mouse-adapted SARS-CoV-2 infection model to explore potential therapeutic targets for SARS-CoV-2 pneumonia. Global gene expression analysis of infected mouse lungs revealed dysregulation of genes associated with NAD+ metabolism, immune response and cell death, similar to that in COVID-19 patients. We therefore investigated the effect of treatment with NAD+ and found that the pneumonia phenotypes, including excessive inflammatory cell infiltration and embolization in SARS-CoV-2 infected lungs were significantly rescued by boosting NAD+ levels. Most notable, cell death was suppressed substantially ( $>65 \%)$ by NAD+ supplementation. Thus, our in vivo mouse study supports trials for treating COVID-19 patients with NAD+ or its precursors.

\section{Main Text}

Novel severe acute respiratory syndrome coronavirus 2 (SARS-CoV-2) has caused the global epidemic of coronavirus disease 2019 (COVID-19) which started in China around December 2019. Patients with critical COVID-19 develop severe respiratory distress, and death (Pan et al., 2020). The ongoing COVID-19 pandemic in the absence of an effective vaccine has prioritized the development of drugs for COVID-19 treatment.

To explore potential therapeutic targets for COVID-19, we inspected the dysregulated genes in a mouseadapted SARS-CoV-2 (MASCp6) infection mouse model (Gu et al., 2020). We performed global gene expression analysis of SARS-CoV-2 infected lungs of 8-month old mice 3.5 days post infection (dpi). GO and KEGG gene set enrichment analysis of significantly up-regulated genes revealed the strong activation of immune related biological processes and pathways, including cytokine-cytokine receptor interaction, NOD-like receptor signaling, chemokine signaling, IL-17 signaling, TNF signaling, IL-6 production and other virus defense pathways (Figure S1A, B). Infection led to robust induction of chemokine and interleukin genes, including I/1b, I/6, Ccl2, Ccl3, Ccl4, Ccl7, Cxcl1, Cxcl2, Cxcl5, Cxc/9, and Cxc/10, as well as many related receptor genes, including $C c r 1, C c r 4, C c r 5, C c r 5, C c r 7, C x c r 2, C x c r 5$, II1r2, and I/1rn (Figure S1B, C; Figure S2A). In addition, 20 genes associated with necroptosis and apoptosis, such as Tnf, T/r3, Daxx, Stat1 and Stat2 were significantly up-regulated (Figure S2B). Moreover, neutrophils, macrophages, dendritic cells, $B$ cells, and $C D 4^{+} \mathrm{T}$ cells related genes were all up-regulated in infected mice as was also observed in COVID-19 patients (Figure S2C) (Bost et al., 2020). These results indicated that SARS-CoV-2 infection induces very similar immune processes in both mice and humans (Bost et al., 2020; Chua et al., 2020; Liao et al., 2020; Zhu et al., 2020)

Nicotinamide adenine dinucleotide $\left(\mathrm{NAD}^{+}\right)$, the cell hydrogen carrier for redox enzymes, plays important roles in multiple metabolic pathways (Miller et al., 2020; Nasi et al., 2020; Omran and Almaliki, 2020). We recently found that PARPs, one of main NAD+-responsive signaling family, were significantly up-regulated while $\mathrm{NAD}^{+}$levels were substantially reduced in Zika virus infected mouse brains (Pang et 
al., manuscript submitted). In SARS-CoV-2 infected lungs, Parp9, Parp10, and Parp14, the three PARPs members which regulate immune and inflammatory responses (Rajman et al., 2018), were also significantly up-regulated (Figure $1 \mathrm{~A}$ ). Meanwhile, $\mathrm{NAD}^{+}$-responsive genes, the Sirtuin family members including Sirt2 and Sirt4, were both down-regulated (Figure S2D). We therefore explored the expression of genes involved in $\mathrm{NAD}^{+}$biosynthetic process and found some of those required for $\mathrm{NAD}^{+}$de novo synthesis and the kynurenine pathway from tryptophan, were up-regulated (Figure 1B, C). In addition, the expression of Nampt, one of the key enzymes for $\mathrm{NAD}^{+}$salvage was induced (Figure 1B). The above results suggested that over-consumption of $\mathrm{NAD}^{+}$by up-regulation of PARPs led to decreased NAD levels and this stimulated compensatory de novo synthesis and salvaging of $N A D^{+}$in infected lungs.

Based on the observations that both SARS-CoV-2 and ZIKV infection led to dysregulation of NAD ${ }^{+}$ associated genes (Figure1 and $\mathrm{S} 2$ ) and reduction of $\mathrm{NAD}^{+}$levels in both mice and human sera (our study and (Heer et al., 2020), we investigated whether NAD ${ }^{+}$supplementation could rescue SARS-CoV-2-induced pathological effects. We first characterized in more detail the pathological features (pneumonia) of infected mouse lungs. Immunostaining with SARS-CoV-2 spike protein and nucleocapsid protein antibodies indicated that SARS-CoV-2 mainly infected airway epithelial cells and some alveolar epithelial cells closed to airways (Figure S3). The infection pattern is similar to another mouse-adapted model of SARS-CoV-2, in which receptor binding domain in the spike protein was remodeled to facilitate its efficient binding to mouse ACE2 (Dinnon et al., 2020). We confirmed the presence of inflammatory cell infiltration, alveolar septal thickening, embolization, epithelial damage, and cell death in MASCp6 infected lungs, similar to the effects in the hACE2 mouse model (Figure 2A; Figure S4A, B) (Jiang et al., 2020; Sun et al., 2020). Notably, we detected embolization in some airways with many macrophages (CD68 $\left.{ }^{+}\right)$ phagocytizing infected cells (Figure S4A). We treated SARS-CoV-2 infected mice with NAD ${ }^{+}(1 \mathrm{mg} / \mathrm{g} /$ day i.p. for 3 days), and sacrificed them on $3.5 \mathrm{dpi}$ (Figure S5A). Compared with severe inflammatory cell infiltration and embolization of airway detected by H\&E staining in saline group, the number and size of inflammatory cell aggregations were significantly reduced obviously in the $\mathrm{NAD}^{+}$treatment group (Figure $2 A-C)$. In addition, the ratio of embolization airway and the relative embolism area were both reduced very significantly $(P<0.001)$ after treatment (Figure 2D, E; Figure S5B). However, comparable levels of SARSCoV-2 intensity and infected area were detected in saline and $\mathrm{NAD}^{+}$treatment groups (Figure S5B-E) suggesting the $\mathrm{NAD}^{+}$treatment did not reduce the rate of infection but relieved its symptoms.

Previous studies have shown that SARS-CoV-2 infection induce the infiltration of neutrophils and macrophages in lungs of patients (Bao et al., 2020; Sun et al., 2020). We found there was more severe aggregation of macrophages in the small airways, especially in those that were close to alveoli, than the big airways in mouse lungs (Figure 3A). The numbers of macrophages and epithelial cells positive for the activated form of caspase 3 was reduced very significantly $(>65 \%)$ in NAD ${ }^{+}$treated mice (Figure 3; Figure S6D, E). Meanwhile, there were much fewer infected alveolar epithelial cells close to the small airways in $\mathrm{NAD}^{+}$treated mice (Figure S5B). These results indicate that $\mathrm{NAD}^{+}$supplementation can protect the lung 
from inflammatory injury, including cell death, caused by SARS-Cov-2 infection in both old and young mice (Figure S6).

In this study, we demonstrated that very similar immune processes and pathways, including cytokine-cytokine receptor interaction, TNF signaling, and IL-6 production, were activated in SARS-CoV-2 infected mice as those observed in SARS-CoV-2 infected patients and organoids model (Bost et al., 2020; Liao et al., 2020; Yang et al., 2020; Zhu et al., 2020). A previous clinical study indicated that IL-6 monoclonal antibody, tocilizumab, could improve the outcome in severe and critical COVID-19 patients (Xu et al., 2020). Together with the presence of typical pneumonia phenotypes in infected lungs, we can conclude that the mouse model is suitable for drug development before clinical trials in COVID-19 patients.

Consistent with our work, a previous metabolomics study showed that the level of tryptophan, the major precursor for the $\mathrm{NAD}^{+}$de novo synthesis pathway, was decreased, and the levels of intermediate substrates, kynurenine and quinolinate, were increased in the sera from severe COVID-19 patients (Shen et al., 2020). We have revealed here that these changes likely reflect dysregulated the $N A D^{+}$related gene set by SARS-Cov-2 infection and induced expression of a set of PARP family members, similar to those in COVID-19 patients (Heer et al., 2020).

Recently, $\mathrm{NAD}^{+}$has been proposed as a selective inhibitor for both the SARS-CoV-2 main protease $\left(\mathrm{M}^{\mathrm{pro}}\right.$ ) and multi-functional papain-like protease ( $\mathrm{PL}^{\text {pro }}$ ) (Kandeel et al., 2020; Martorana et al., 2020). However, our results indicate that $\mathrm{NAD}^{+}$supplementation leads to limited area of infection but does not inhibit multiplication of SARS-CoV-2 in vivo. We found instead that NAD ${ }^{+}$could effectively prevent the cell death caused by SARS-CoV-2 and ZIKV in mouse lung and brain, respectively (this study and other manuscript submitted). Based on the similar dysregulation of $\mathrm{NAD}^{+}$metabolism in both ZIKV and SARSCoV-2 infected mouse models, the mechanism underlying treatment with $\mathrm{NAD}^{+}$for cell death may be similar. NAD ${ }^{+}$deficiency induced by up-regulated PARPs could affect the function of many related metabolic pathways, such as oxidative phosphorylation, TCA cycle, and tryptophan metabolism. NAD ${ }^{+}$ supplementation helps to maintain the balance of metabolic processes in virus infected cells and prevent the death of those cells, as well as suppress the subsequent inflammatory response. This may explain why NAD ${ }^{+}$precursors, including NMN, NR and NAM, represent anti-inflammatory effects in different animal models including aging, autoimmune encephalomyelitis, ischemia, Ataxia Telangiectasia (Fang et al., 2016; Gomes et al., 2013; Kaneko et al., 2006; Katsyuba et al., 2020; Rajman et al., 2018). Therefore, our study is in strong support of initiating a trial for treating COVID-19 patients with NAD ${ }^{+}$or its precursors.

\section{Declarations}

\section{AUTHOR CONTRIBUTIONS}


Z.X. and C.-F.Q. conceived of the research and Z.X. designed the study. Z.X. and Y.J. wrote the manuscript. Y.J. and Y.D. designed and performed most of the experiments. Y.J. did RNA sequencing analysis and immunostaining. Y.D. did inoculation of the virus and took care of the mice. T.M. did H\&E staining. P.H and Z.H. contributed to the ideas and writing of the paper as everyone else.

\section{ACKNOWLEDGMENTS}

We thank Dr. John Speakman for his kind comments and editing of the manuscript. This study was supported by grants from the Strategic Priority Research Program and Innovation Program of the Chinese Academy of Sciences (XDB32020100, QYZDJ-SSW-SMC007), and National Natural Science Foundation of China (NSFC) $(31921002,31730108)$ to Z Xu.

\section{Key Resources}

Key Resources 


\begin{tabular}{|c|c|c|}
\hline REAGENT or RESOURCE & SOURCE & IDENTIFIER \\
\hline \multicolumn{3}{|l|}{ Experimental animal } \\
\hline BALB/c mice & $\begin{array}{l}\text { Beijing Vital River } \\
\text { Animal Technology }\end{array}$ & $\mathrm{N} / \mathrm{A}$ \\
\hline \multicolumn{3}{|l|}{ Antibodies } \\
\hline CD68 & Abcam & Cat\#ab125212 \\
\hline Cleaved Caspase 3 & Cell Signaling Technology & Cat\#9664 \\
\hline SARS-CoV-2 spike & Sino Biological & Cat\#40150-D001 \\
\hline SARS-CoV-2 nucleocapsid & Sino Biological & Cat\#40588-T62 \\
\hline DAPI & Cell Signaling Technology & Cat\#4083S \\
\hline \multicolumn{3}{|l|}{ Virus Strains } \\
\hline SARS-CoV-19 & Dr. Yusen Zhou and Dr. Cheng-Feng Qin & MASCp6 \\
\hline \multicolumn{3}{|l|}{ Biological Samples } \\
\hline Lung samples of 6-7 weeks old BALB/c mice & This study & $\mathrm{N} / \mathrm{A}$ \\
\hline Lung samples of 8 months old BALB/c mice & This study & $\mathrm{N} / \mathrm{A}$ \\
\hline \multicolumn{3}{|l|}{ Chemicals } \\
\hline $\mathrm{NAD}^{+}$ & Sigma & Cat\#N7004 \\
\hline TRIzol & Life technologies & REF\#15596018 \\
\hline \multicolumn{3}{|l|}{ Histopathology } \\
\hline Hematoxylin-Eosin/HE Staining Kit & Solarbio & Cat\#G1120 \\
\hline \multicolumn{3}{|l|}{ Software and Algorithms } \\
\hline \multicolumn{3}{|l|}{ ImageJ (1.48v) } \\
\hline \multicolumn{3}{|l|}{ GraphPad (Prism 7) } \\
\hline ZEN3.1 (blue edition) & ZEISS & \\
\hline \multicolumn{3}{|l|}{ Cytoscape (version 3.7.2) } \\
\hline \multicolumn{3}{|l|}{ R and RStudio (version 3.5.1) } \\
\hline BioRender & & https://biorender.com/ \\
\hline \multicolumn{3}{|l|}{ Deposited Data } \\
\hline RNA-seq data & This study & \\
\hline
\end{tabular}

\section{Experimental Model And Subject Details}

\section{Ethics statement}

All procedures involving animals were conducted in temperature- and humidity-controlled Biosafety Level 3 laboratory (BSL-3) and approved by the Animal Experiment Committee of Laboratory Animal Center, Beijing Institute of Microbiology and Epidemiology (approval number: IACUC-DWZX-2020-002). All animal 
experiments were handled in accordance with the recommendations in the Guide for the Care and Use of Laboratory Animals.

\section{Mice}

All BALB/c mice were bought from Bejing Vital River Laboratory Animal Technology Co., Ltd. Female, 6-7 weeks old and 8 months old mice were used for all experiments.

\section{Virus Strain}

SARS-CoV-2 strains MASCp6 (mouse-adapted SARSCoV-2 at passage 6) was obtained from Dr. Yusen Zhou and Dr. Cheng-Feng Qin. All experiments involving infectious SARS-CoV-2 were performed in biosafety level 3 (BSL-3) containment laboratory in Beijing Institute of Microbiology and Epidemiology.

\section{Method Details}

\section{Infection and administration of BALB/c mice.}

Anesthetized 6-7 weeks and 8 months old female BALB/c mice were intranasally (i.n.) treated with a dose of $7.2 \times 10^{5}$ plaque forming unit (PFU) of SARS-CoV-2 in a total volume of $40 \mu \mathrm{L}$ in each group. The infected mice were administered with either $\mathrm{NAD}^{+}$dissolved in $0.9 \%$ saline or the equivalent volume of $0.9 \%$ saline for three consecutive days and one time per day by intraperitoneal injection starting at half a day after inoculation the virus. These procedures were operated in Biosafety Level 3 laboratory (BSL-3).

\section{Collection of mice samples}

The mice were euthanized on $3.5 \mathrm{dpi}$. The lungs and tracheas of each mouse were removed and fixed in $4 \%$ paraformaldehyde (PFA) at $4^{\circ} \mathrm{C}$ two days for frozen section and paraffin section or homogenized with TRIzol reagent for RNA extraction.

\section{Measurement of viral RNA}

Lung and trachea tissue homogenates were clarified by centrifugation at 6,000 rpm for $6 \mathrm{~min}$, and the supernatants were transferred to a new EP tube. Viral RNA (VRNA) was extracted using the QIAamp Viral RNA Mini Kit (Qiagen, Cat\#52906) according to the manufacturer's protocol. vRNA quantification in each 
sample was performed by Q-PCR targeting the S gene of SARS-CoV-2 as described previously (Gu et al., 2020).

\section{Histopathological staining}

Fixed lung tissues for histological examination were subjected to alcohol gradient dehydration and embedded in paraffin. Each embedded tissue was sectioned at $5 \mu \mathrm{m}$ thickness sections. At least three sections of each tissue were used for staining with hematoxylin and eosin (H\&E) and examined under a light microscopy. The tissue handling and H\&E staining were accorded to standard protocol.

\section{Immunofluorescent staining}

4\% PFA fixed lung tissues were dehydrated in 30\% sucrose for 2 days and embedded in optimal cutting temperature compound (O.C.T. Compound, SAKURA, REF4583, USA). $25 \mu \mathrm{m}$ sections were sliced with freezing microtome (CM 1950, Leica, Germany). Sections were blocked with blocking buffer (PBS $+10 \%$ $\mathrm{FBS}+3 \% \mathrm{BSA}+0.2 \%$ TrintonX100) at room temperature for 1 hour and incubated with the primary antibodies at $37{ }^{\circ} \mathrm{C}$ for 2 hours. After washing with PBST (PBS+0.2\% TrintonX100) for 3 times (10 min for each time), the lung sections were subsequently incubated with fluorescent secondary antibodies at room temperature for $1 \mathrm{~h}$, followed by washing with PBST for 3 times (10min for each time). Primary antibodies include: rabbit anti-Cleaved Caspase 3 (1:500, Cell Signaling Technology, Cat\#9664), rabbit anti-CD68 (1:1000, Abcam, Cat\#ab125212), rabbit anti-SARS-CoV-19 nucleocapsid (1:100, Sino Biological, Cat\#40588-T62), human/mouse anti-SARS-CoV-19 spike (1:100, Sino Biological, Cat\#40150-D001). Secondary antibodies: AlexaFluor-488-, AlexaFluor-568-, and AlexaFluor-647-conjugated goat secondary antibodies against rabbit and human IgG (1:1000; Invitrogen). Nuclei were counterstained with DAPI (Cell Signaling Technology, Cat\#4083s). Fluorescent images were captured using a confocal laser-scanning microscope (Carl Zeiss, LSM700) and analyzed with ZEN3.1 and ImageJ.

\section{RNA extraction and sequencing}

Total RNA from the lung homogenates was extracted with TRIzol reagent and sent to LC-BIO TECHNOLOGIES (HANGZHOU) for sequencing and analysis. Purified RNA was performed the $2 \times 150 \mathrm{bp}$ paired-end sequencing (PE150) on an Illumina Novaseq ${ }^{\text {TM }} 6000$ (LC-Bio Technology CO., Ltd., Hangzhou, China) following the vendor's recommended protocol. After preprocessing, the high-quality reads were compared with the mouse reference genome (ftp://ftp.ensembl.org/pub/release99/fasta/mus_musculus/dna/) by HISAT2 software (https://daehwankimlab.github.io/hisat2/). 


\section{RNA-seq analysis}

The significantly differentially expressed mRNAs were selected with $\mid \log _{2}$ (fold change) $\mid>1$ and $p$ value < 0.05 by R package DESeq2 (Pertea et al., 2015). GO enrichment analysis and KEGG enrichment analysis of significantly differently expressed genes were performed using the OmicStudio tools at https://www.omicstudio.cn/tool. Gene number and $p$ value of GO and KEGG terms were visualized using the $\mathrm{R}$ package ggplot2. The network of the genes among different $\mathrm{GO}$ and KEGG terms were visualized using Cytoscape (version 3.7.2). The heat maps and bar plots of significantly differently expressed genes were drawn with the $\mathrm{R}$ package ggplot2 and GraphPad (Prism 7) respectively.

\section{Quantification And Statistical Analysis}

For quantification of inflammatory cell aggregation number and area, embolism airway ratio and relative area, and alveolar septum relative area, three lung sections for each mouse (3 mice for saline group and 4 mice for $\mathrm{NAD}^{+}$group) were included. Inflammatory cell aggregation was defined by dense cell cluster and dark blue staining with hematoxylin. The density of aggregation was calculated as the number of aggregation divided by the area of minimal convex polygon which can cover all of aggregation in each lung section. The area of aggregation was the total area added by area of each individual aggregation. Embolism airway ratio was calculated as the number of airways having embolism divided by the number of total airways in each section. The relative area of embolism was calculated as the area of each embolism divided by the area of corresponding airway. The relative area of alveolar septum was calculated as the total area of the substances divided by the area of whole lung section. For quantification of the density of $\mathrm{CD} 68^{+}$cells, five regions for each lung section, two sections for each mouse were included. The density was calculated as the number of $\mathrm{CD} 68^{+}$cells divided by the area of corresponding region. For quantification of the ratio of $\mathrm{Cas}^{+}$cells to infected cells, three to four infected airways for each mouse were included. The ratio was calculated as the number of $\mathrm{Cas}^{+}{ }^{+}$cells divided by the number of infected cells in the same location of the adjacent section. For quantification of SARS-CoV2 relative fluorescence intensity and infected area, two sections for each mouse were included. Relative fluorescence intensity was calculated as the total fluorescence intensity of SARS-CoV-2 signal divided by the area of whole lung section. The relative infected area was calculated as the area of minimal convex polygon which can cover all of virus signal divided as the area of whole lung section. Statistical analyses were carried out with unpaired $t$ test or Welch's $t$ test. Data are presented as mean \pm SEM.

\section{References}

Bao, L., Deng, W., Huang, B., Gao, H., Liu, J., Ren, L., Wei, Q., Yu, P., Xu, Y., Qi, F., et al. (2020). The pathogenicity of SARS-CoV-2 in hACE2 transgenic mice. Nature 583, 830-833. 
Bost, P., Giladi, A., Liu, Y., Bendjelal, Y., Xu, G., David, E., Blecher-Gonen, R., Cohen, M., Medaglia, C., Li, H., et al. (2020). Host-Viral Infection Maps Reveal Signatures of Severe COVID-19 Patients. Cell 181, 1475-1488 e1412.

Chua, R.L., Lukassen, S., Trump, S., Hennig, B.P., Wendisch, D., Pott, F., Debnath, O., Thurmann, L., Kurth, F., Volker, M.T., et al. (2020). COVID-19 severity correlates with airway epithelium-immune cell interactions identified by single-cell analysis. Nat Biotechnol.

Dinnon, K.H., 3rd, Leist, S.R., Schafer, A., Edwards, C.E., Martinez, D.R., Montgomery, S.A., West, A., Yount, B.L., Jr., Hou, Y.J., Adams, L.E., et al. (2020). A mouse-adapted model of SARS-CoV-2 to test COVID-19 countermeasures. Nature.

Fang, E.F., Kassahun, H., Croteau, D.L., Scheibye-Knudsen, M., Marosi, K., Lu, H., Shamanna, R.A., Kalyanasundaram, S., Bollineni, R.C., Wilson, M.A., et al. (2016). NAD(+) Replenishment Improves Lifespan and Healthspan in Ataxia Telangiectasia Models via Mitophagy and DNA Repair. Cell Metab 24, 566-581.

Gomes, A.P., Price, N.L., Ling, A.J., Moslehi, J.J., Montgomery, M.K., Rajman, L., White, J.P., Teodoro, J.S., Wrann, C.D., Hubbard, B.P., et al. (2013). Declining NAD(+) induces a pseudohypoxic state disrupting nuclear-mitochondrial communication during aging. Cell 155, 1624-1638.

Gu, H., Chen, Q., Yang, G., He, L., Fan, H., Deng, Y.Q., Wang, Y., Teng, Y., Zhao, Z., Cui, Y., et al. (2020). Adaptation of SARS-CoV-2 in BALB/c mice for testing vaccine efficacy. Science.

Heer, C.D., Sanderson, D.J., Alhammad, Y.M.O., Schmidt, M.S., Trammell, S.A.J., Perlman, S., Cohen, M.S., Fehr, A.R., and Brenner, C. (2020). Coronavirus and PARP expression dysregulate the NAD Metabolome: a potentially actionable component of innate immunity. bioRxiv.

Jiang, R.D., Liu, M.Q., Chen, Y., Shan, C., Zhou, Y.W., Shen, X.R., Li, Q., Zhang, L., Zhu, Y., Si, H.R., et al. (2020). Pathogenesis of SARS-CoV-2 in Transgenic Mice Expressing Human Angiotensin-Converting Enzyme 2. Cell 182, 50-58 e58.

Kandeel, M., Abdelrahman, A.H.M., Oh-Hashi, K., Ibrahim, A., Venugopala, K.N., Morsy, M.A., and Ibrahim, M.A.A. (2020). Repurposing of FDA-approved antivirals, antibiotics, anthelmintics, antioxidants, and cell protectives against SARS-CoV-2 papain-like protease. J Biomol Struct Dyn, 1-8.

Kaneko, S., Wang, J., Kaneko, M., Yiu, G., Hurrell, J.M., Chitnis, T., Khoury, S.J., and He, Z. (2006). Protecting axonal degeneration by increasing nicotinamide adenine dinucleotide levels in experimental autoimmune encephalomyelitis models. J Neurosci 26, 9794-9804.

Katsyuba, E., Romani, M., Hofer, D., and Auwerx, J. (2020). NAD(+) homeostasis in health and disease. Nat Metab 2, 9-31. 
Liao, M., Liu, Y., Yuan, J., Wen, Y., Xu, G., Zhao, J., Cheng, L., Li, J., Wang, X., Wang, F., et al. (2020). Singlecell landscape of bronchoalveolar immune cells in patients with COVID-19. Nat Med 26, 842-844.

Martorana, A., Gentile, C., and Lauria, A. (2020). In Silico Insights into the SARS CoV-2 Main Protease Suggest NADH Endogenous Defences in the Control of the Pandemic Coronavirus Infection. Viruses 12.

Miller, R., Wentzel, A.R., and Richards, G.A. (2020). COVID-19: NAD(+) deficiency may predispose the aged, obese and type2 diabetics to mortality through its effect on SIRT1 activity. Med Hypotheses 144, 110044.

Nasi, A., McArdle, S., Gaudernack, G., Westman, G., Melief, C., Rockberg, J., Arens, R., Kouretas, D., Sjolin, J., and Mangsbo, S. (2020). Reactive oxygen species as an initiator of toxic innate immune responses in retort to SARS-CoV-2 in an ageing population, consider $\mathrm{N}$-acetylcysteine as early therapeutic intervention. Toxicol Rep 7, 768-771.

Omran, H.M., and Almaliki, M.S. (2020). Influence of NAD+ as an ageing-related immunomodulator on COVID 19 infection: A hypothesis. J Infect Public Health.

Pan, F., Ye, T., Sun, P., Gui, S., Liang, B., Li, L., Zheng, D., Wang, J., Hesketh, R.L., Yang, L., et al. (2020). Time Course of Lung Changes at Chest CT during Recovery from Coronavirus Disease 2019 (COVID-19). Radiology 295, 715-721.

Pertea, M., Pertea, G.M., Antonescu, C.M., Chang, T.C., Mendell, J.T., and Salzberg, S.L. (2015). StringTie enables improved reconstruction of a transcriptome from RNA-seq reads. Nat Biotechnol 33, 290-295.

Rajman, L., Chwalek, K., and Sinclair, D.A. (2018). Therapeutic Potential of NAD-Boosting Molecules: The In Vivo Evidence. Cell Metab 27, 529-547.

Shen, B., Yi, X., Sun, Y., Bi, X., Du, J., Zhang, C., Quan, S., Zhang, F., Sun, R., Qian, L., et al. (2020). Proteomic and Metabolomic Characterization of COVID-19 Patient Sera. Cell 182, 59-72 e15.

Sun, S.H., Chen, Q., Gu, H.J., Yang, G., Wang, Y.X., Huang, X.Y., Liu, S.S., Zhang, N.N., Li, X.F., Xiong, R., et al. (2020). A Mouse Model of SARS-CoV-2 Infection and Pathogenesis. Cell Host Microbe 28, 124-133 e124.

Xu, X., Han, M., Li, T., Sun, W., Wang, D., Fu, B., Zhou, Y., Zheng, X., Yang, Y., Li, X., et al. (2020). Effective treatment of severe COVID-19 patients with tocilizumab. Proc Natl Acad Sci U S A 117, 10970-10975.

Yang, L., Han, Y., Nilsson-Payant, B.E., Gupta, V., Wang, P., Duan, X., Tang, X., Zhu, J., Zhao, Z., Jaffre, F., et al. (2020). A Human Pluripotent Stem Cell-based Platform to Study SARS-CoV-2 Tropism and Model Virus Infection in Human Cells and Organoids. Cell Stem Cell 27, 125-136 e127.

Zhu, L., Yang, P., Zhao, Y., Zhuang, Z., Wang, Z., Song, R., Zhang, J., Liu, C., Gao, Q., Xu, Q., et al. (2020). Single-Cell Sequencing of Peripheral Mononuclear Cells Reveals Distinct Immune Response Landscapes of COVID-19 and Influenza Patients. Immunity. 


\section{Figures}

A

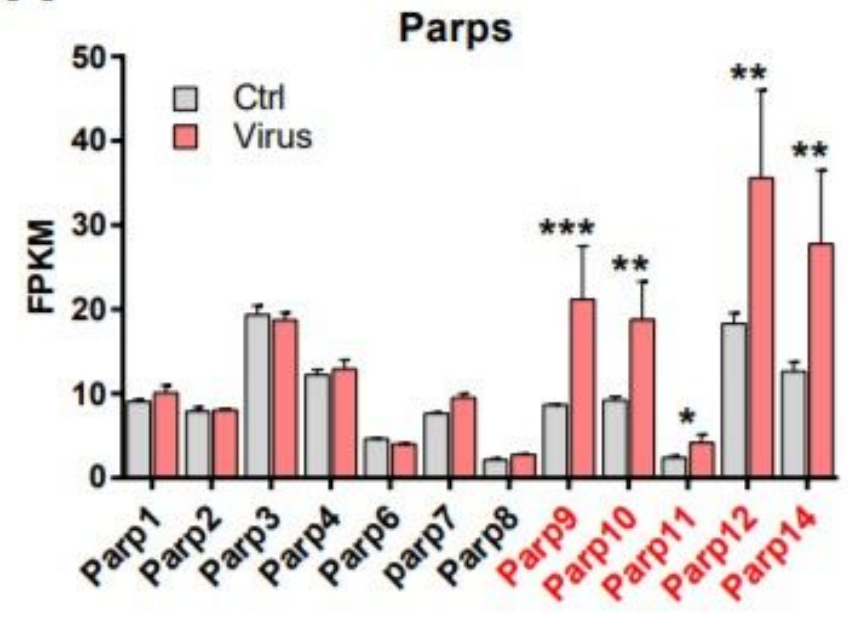

B

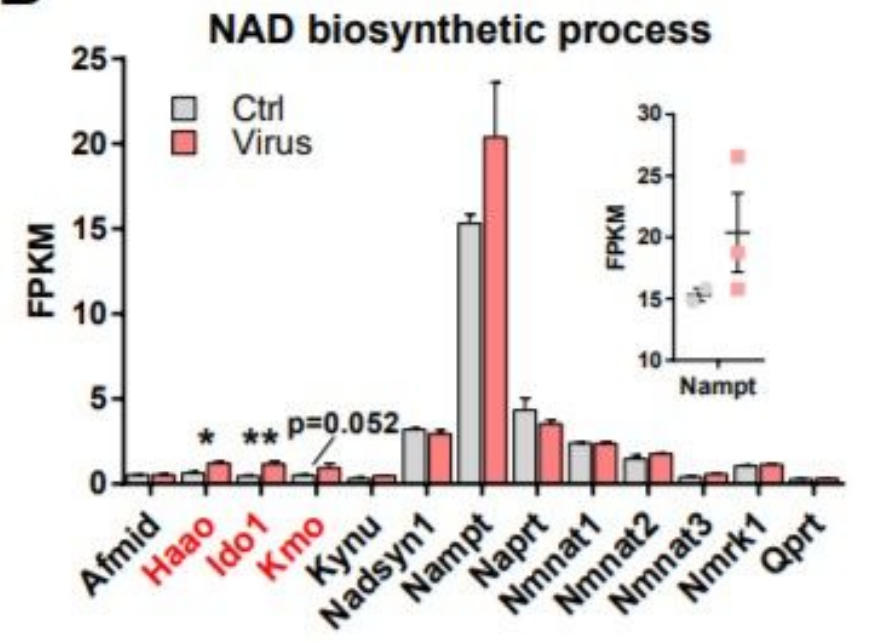

C

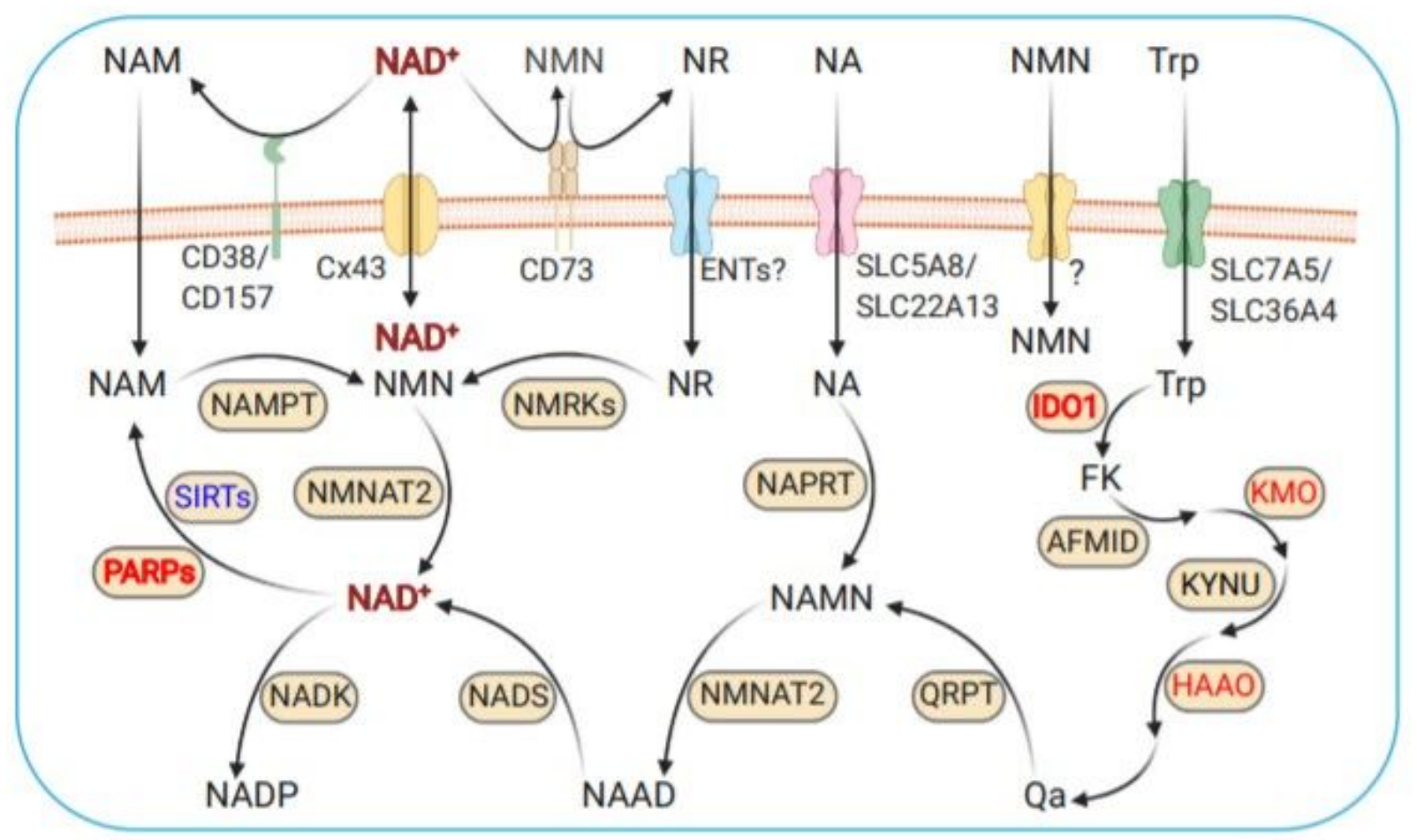

Figure 1

SARS-CoV-2 infection leads to dysregulation of NAD+ associated genes in lungs. (A-B) RNA-seq analysis of SARS-CoV-2 infected lungs of 8-month old mice on 3.5 days post infection (dpi). Bar plots indicate the expression levels of PARPs (A) and NAD+ biosynthetic process related genes (B). All data showed as 
means $\pm S E M,{ }^{*} p<0.05,{ }^{* \star} p<0.01,{ }^{* \star *} p<0.001$. (C) Schema of NAD+ metabolism pathway (Refer to Rajman et al., 2018). NA: nicotinic acid; NAMN: nicotinic acid mononucleotide; NAAD: nicotinic acid adenine dinucleotide; NR: nicotinamide riboside; NAM: nicotinamide; NMN: nicotinamide mononuclotide; Trp: tryptophan; FK: formyl-kynurenine; Qa: quinolinate. Red letters: up-regulated (fold change $>1$ and $p$ value $<0.06$ ); Blue letters: down-regulated (fold change $<1$ and $p$ value $<0.06$ ).

A
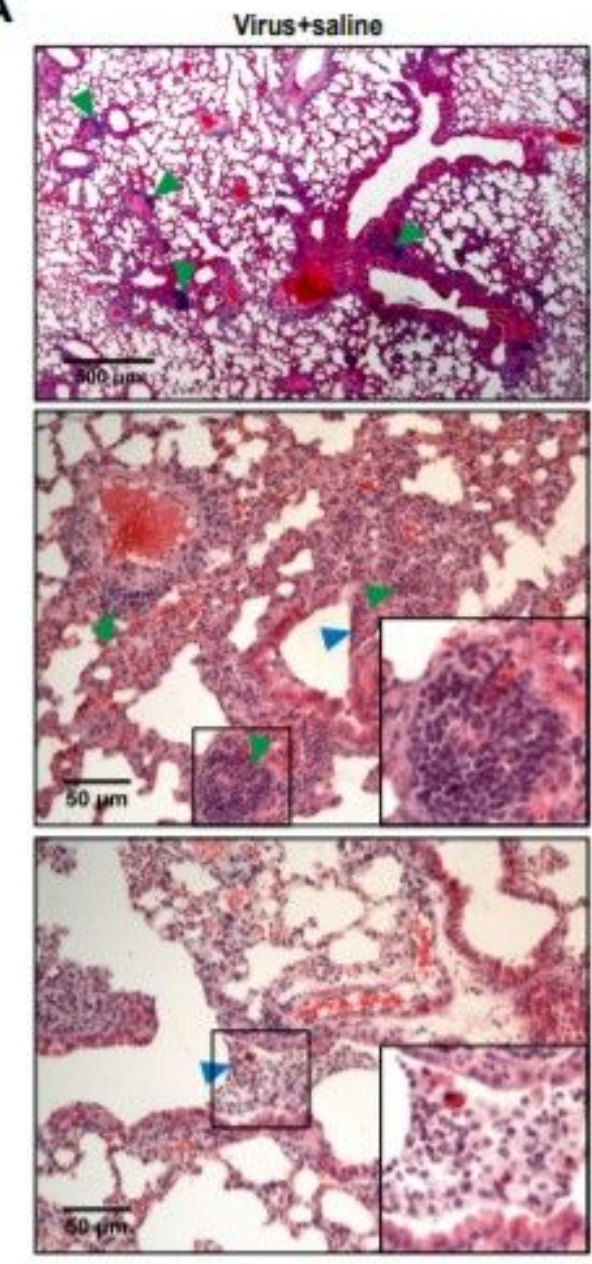

B

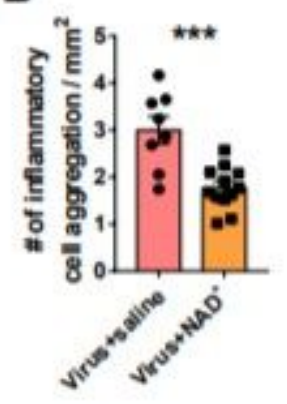

C

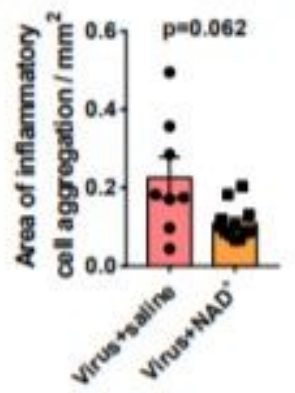

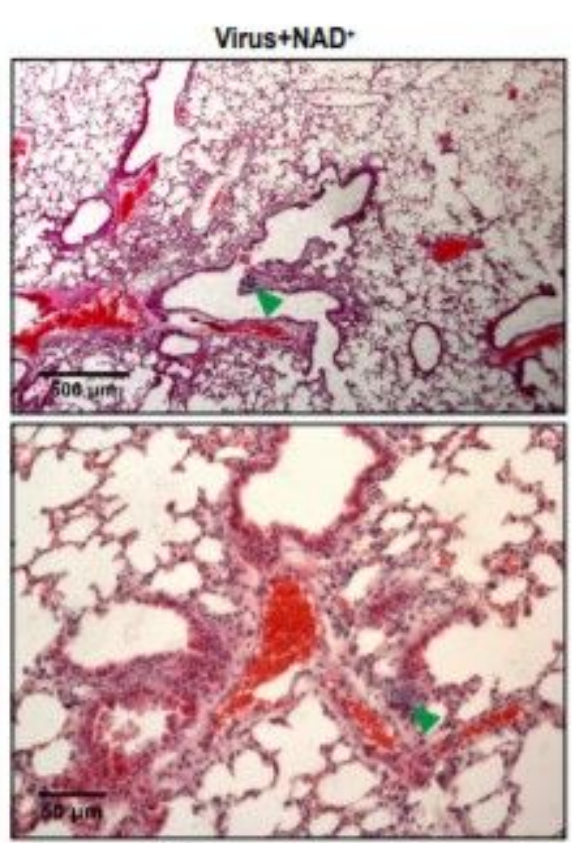

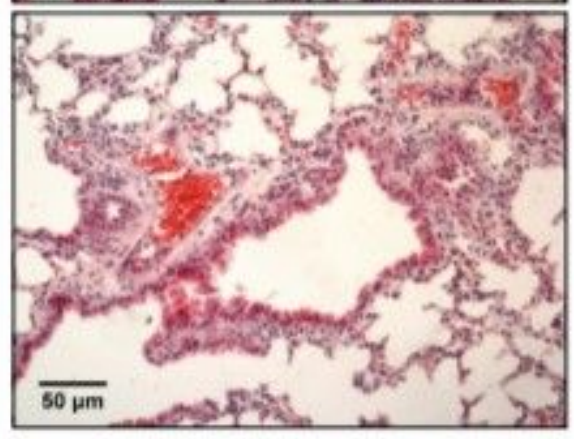

D

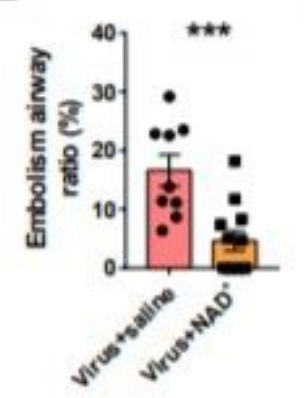

E

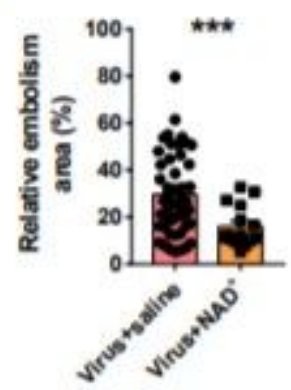

Figure 2

NAD+ supplementation relieves pneumonia caused by SARS-CoV-2 infection. (A) H\&E staining shows that inflammatory cell infiltration (green arrow head) and embolism in airway (blue arrow head) induced 
by infection were rescued by NAD+ supplementation. (B-E) Quantification of the density (B) and area (C) of inflammatory cell aggregation, the ratio of embolism airway (D) and relative area of embolism (E). All data showed as means \pm SEM, $t$ test. ${ }^{*} * \mathrm{p}<0.001$.

A
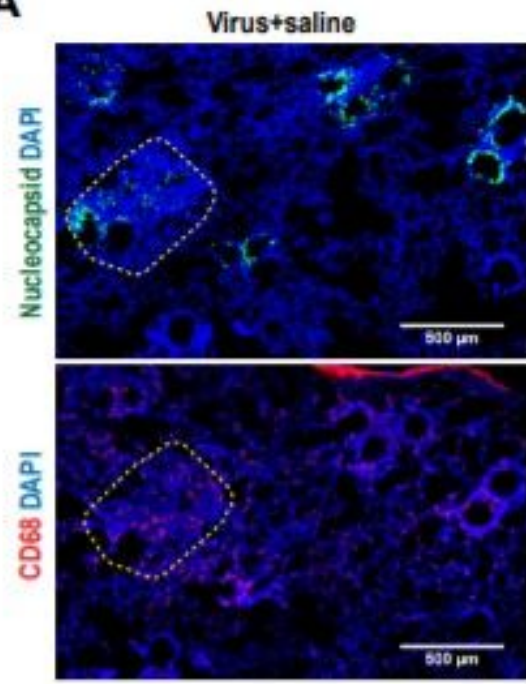

B
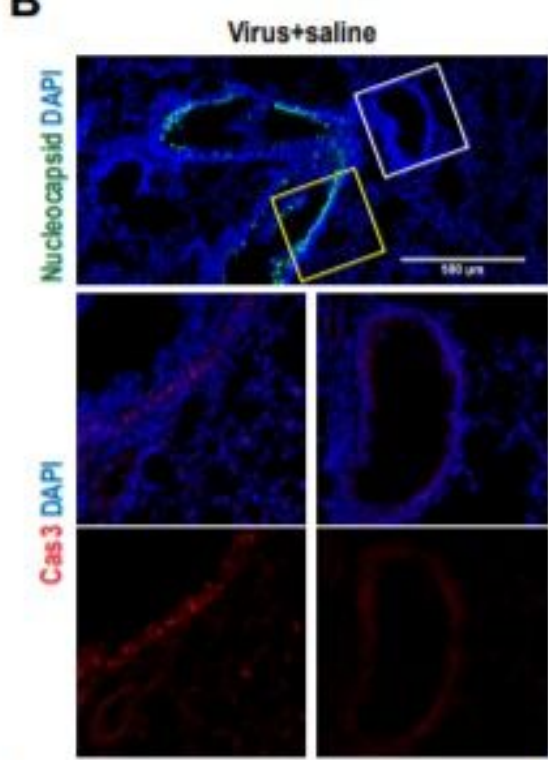

C

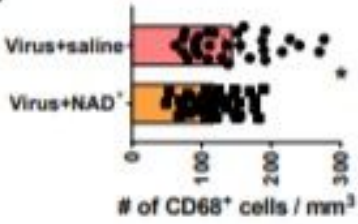

Virus+NAD*

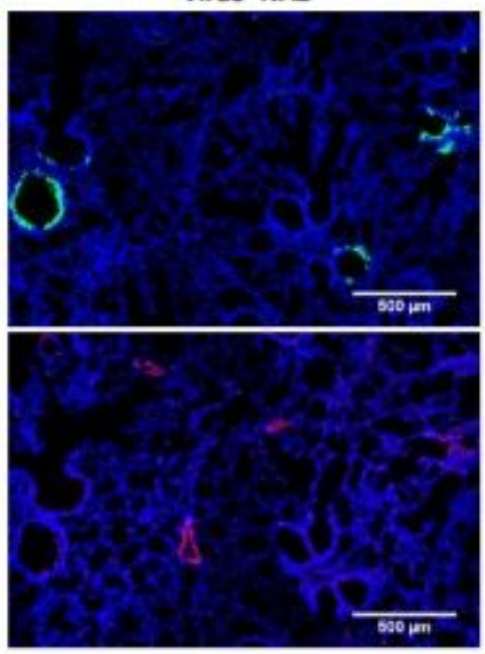

Virus+NAD *
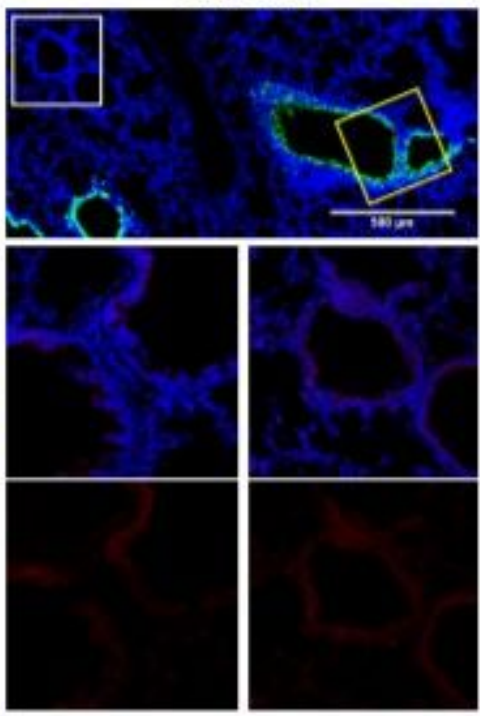

D

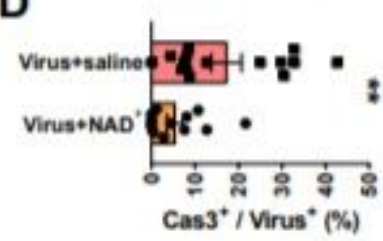

\section{Figure 3}

$N A D+$ supplementation prevents excessive aggregation of macrophages and cell death in infected lungs. (A) Immunostaining of SARS-CoV-2 nucleocapsid protein (top panels, green) and marker of macrophage, CD68 (bottom panels, red) in two adjacent lung sections. Yellow border: excessive aggregation of macrophages. (B) Immunostaining of SARS-CoV-2 nucleocapsid protein (top panels, green) and the 
activated form of Cas3 (middle and bottom panels, red) in two adjacent lung sections. Yellow border: infected airway epithelial cells; White border: airway epithelial cells without virus. (C-D) Quantification of the density of CD68+ cells (C) and the ratio of Cas3+ cells to infected cells (D). All data showed as means \pm SEM, t test. ${ }^{*} p<0.05,{ }^{*} p<0.01$.

\section{Supplementary Files}

This is a list of supplementary files associated with this preprint. Click to download.

- S1.jpg

- S2.jpg

- S3.jpg

- S4.jpg

- S5.jpg

- S6.jpg 